\title{
Novel Prosopis juliflora leaf ethanolic extract as natural antifungal agent against Botrytis cinerea: application on strawberries shelf-life extension
} Iman Saleh

\author{
Department of Biological and Environmental Sciences
}

\section{Supervised by: Professor Mohammed Abu-Dieyeh}

\section{ABSTRACT}

Introduction: Fresh produces' spoilage is a worldwide concern that accompany the global increase in food demand. Adverse human health and environmental effects of commercial pesticides is a major public concern.

Material and Methods: In this report, our Prosopis juliflora water soluble leaves ethanolic (PJ-WS-LE) extract will be described as an effective antifungal agent.

Results: PJ-WS-LE extract showed total inhibition of Botrytis cinerea growth and extend strawberries shelf-life.

Future directions: PJ-WS-LE extract will be chemically described and its effectiveness on other fresh produces will be evaluated.

\section{BACKGROUND}

Botrytis cinerea is one of the top ten pathogens that affect fresh produce shelf-life around the world. With a global effort to control $B$. cinerea grey mold, countries spend a lot of money on botryticides, wide spectrum fungicides and biocontrollers. Botrytis cinerea can progress easily from infected fruits to healthy ones even at low storage temperatures which can lead to entire lots spoilage in few weeks. Prosopis juliflora has been explored for its antimicrobial activities. However, studies are not interconnected.

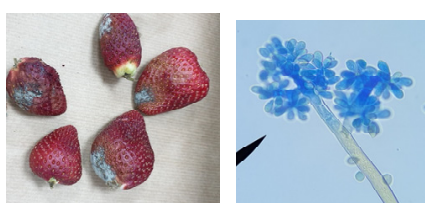

Strawberries are widely consumed raw berries which are famous in their processed forms such as jam and juices. The delicate fruit has a very short postharvest life, it is susceptible to mechanical injuries, fast dehydration and fungal infection. Strawberries' most common spoiling fungi is $B$. cinerea. Our PJ-WS-LE extract is a promising fungicides alternative that showed in-vitro effectiveness against $B$. cinerea.

\section{OBJECTIVES}

- Identifying a natural plant extract bio-controller.

- Controlling $B$. cinerea fungal postharvest spoilage.

- Extending strawberries shelf-life.

- Maintaining strawberries quality parameters during storage.

\section{METHODOLOGY}

4.1 Disk antifungal method

Used to test the effect of $20 \mathrm{mg} / \mathrm{ml}$ of PJ-WS-LE extract in PDA plate on $B$.

Cinerea growth compared to normal growth.

\subsection{Determination of minimum} inhibitory concentration

96-well plate method was used to determine PJ-WS-LE extract MIC against $B$. cinerea.

\subsection{Mode of action}

The inhibited $B$. cinerea disk was transferred to a clean PDA plate to determine the extract mode of action. 4.4 Effect of PJ-WS-LE extract on the microscopic morphology of $B$. cinerea using SEM

Cultures of $B$. cinerea were prepared in the presence $\&$ absence of $8 \mathrm{mg} / \mathrm{ml}$ of PJWS-LE extract. SEM pictures of spores and hyphae were taken.

4.5 Determination of shelf-life of strawberry samples

Treated strawberry samples $(8 \mathrm{mg} / \mathrm{ml} \mathrm{PJ-}$ WS-LE extract) and control samples had their shelf-life determined at room temperature and at $4^{\circ} \mathrm{C}$.

4.6 Strawberries storage quality parameters monitoring

Quality parameters of treated and control samples were monitored on a weekly basis: weight loss, bacterial and fungal CFU, firmness, $\mathrm{pH}$ and TSS.

\section{RESULTS}

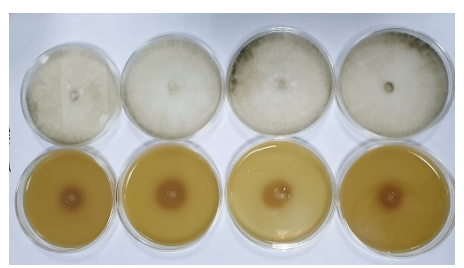

Fig 1. Disk antifungal methods results of B. cinerea

PJ-WS-LE extract has fungicidal effect on $B$. cinerea.

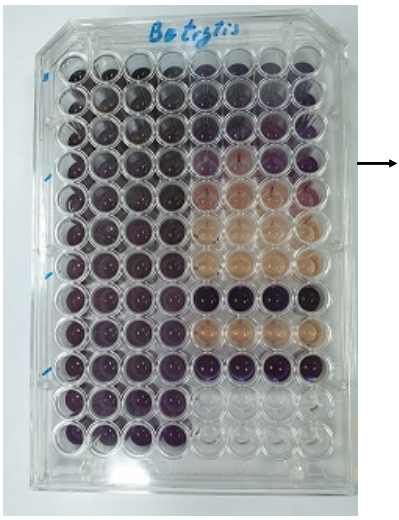

Fig 2. 96-well plate results of $B$. cinerea

\section{NOVOL EXTRACT PREPARATION METHOD}

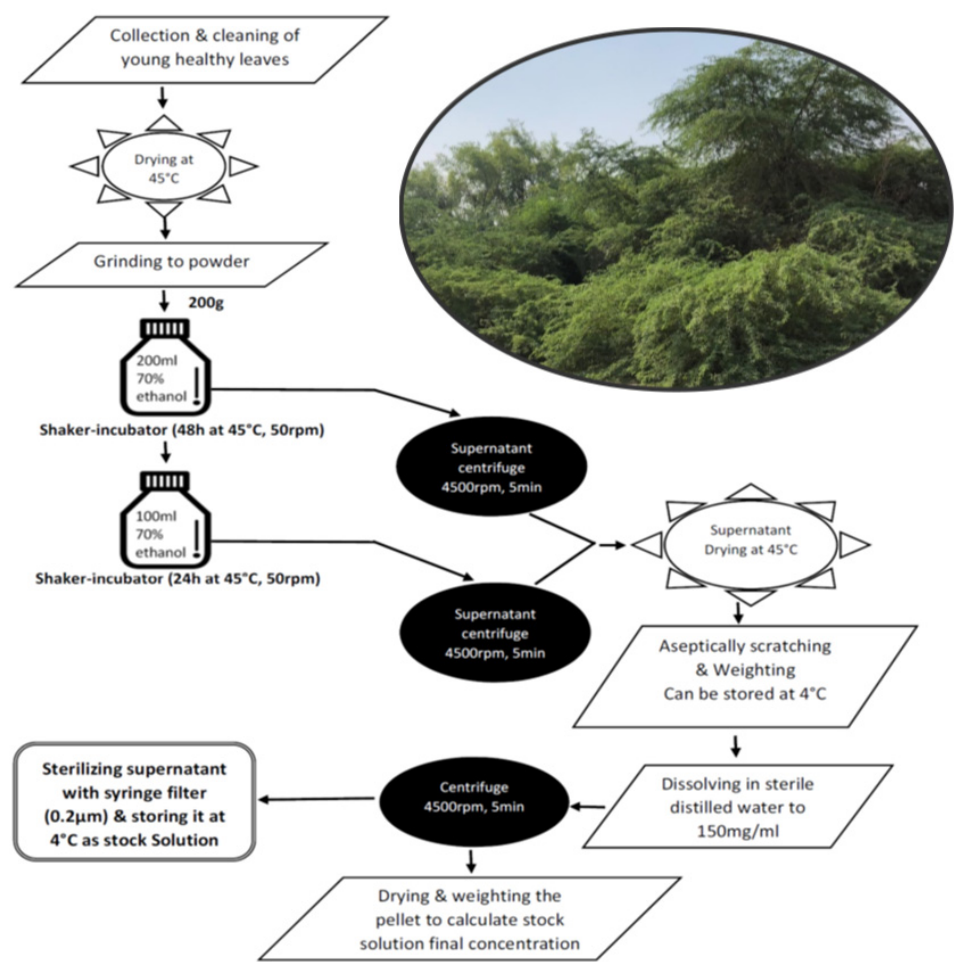

6. RESULTS \& DISCUSSION

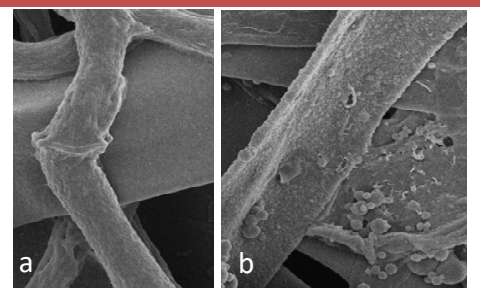

Fig 3. SEM pictures of $B$. cinerea hyphae (control (a)-Treated (b))

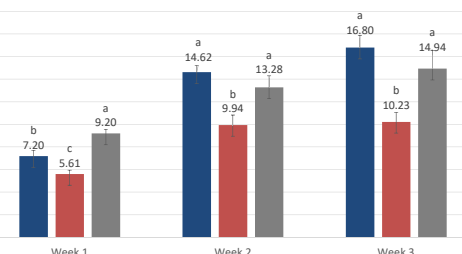

Fig 5. Weekly weight loss percent

(Control (A), coated with 8mg/ml PJ-WS-LE extract (B) coated with $1 \%$ chitosan (C).

\section{CONCLUSIONS}

$>$ PJ-WS-LE totally inhibits the growth of $B$. cinerea with fungicidal activity.

$>$ MIC of PJ-WS-LE extract against $B$. cinerea is $1 \mathrm{mg} / \mathrm{ml}$.

- Coated strawberries $(8 \mathrm{mg} / \mathrm{ml}$ PJ-WS-LE extract) had their shelf-life at $4^{\circ} \mathrm{C}$ extended from 4.3 days to 10 days.

$>8 \mathrm{mg} / \mathrm{ml} \mathrm{PJ}-\mathrm{WS}$-LE extract coating is more effective that $1 \%$ chitosan

$>$ PJ-WS-LE extract showed promising fungicidal characteristics that allow it to replace chemical fungicides.

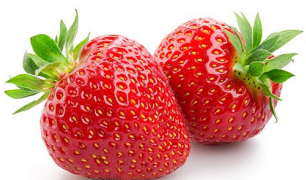

